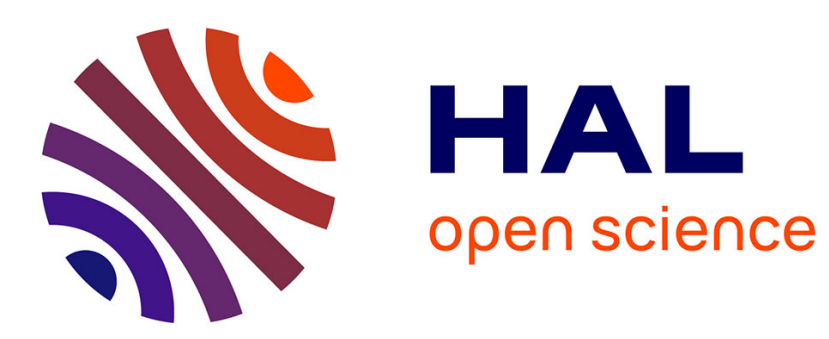

\title{
The Isoconditioning Loci of A Class of Closed-Chain Manipulators
}

\author{
Damien Chablat, Philippe Wenger, Jorge Angeles
}

\section{To cite this version:}

Damien Chablat, Philippe Wenger, Jorge Angeles. The Isoconditioning Loci of A Class of ClosedChain Manipulators. May 1998, Bruxelle, Belgium. pp.1970-1976. hal-00162274

\section{HAL Id: hal-00162274 https://hal.science/hal-00162274}

Submitted on 13 Jul 2007

HAL is a multi-disciplinary open access archive for the deposit and dissemination of scientific research documents, whether they are published or not. The documents may come from teaching and research institutions in France or abroad, or from public or private research centers.
L'archive ouverte pluridisciplinaire $\mathbf{H A L}$, est destinée au dépôt et à la diffusion de documents scientifiques de niveau recherche, publiés ou non, émanant des établissements d'enseignement et de recherche français ou étrangers, des laboratoires publics ou privés. 


\title{
The Isoconditioning Loci of A Class of Closed-Chain Manipulators
}

\author{
Damien Chablat Philippe Wenger Jorge Angeles ${ }^{\mathbf{1}}$ \\ Institut de Recherche en Cybernétique de Nantes \\ École Centrale de Nantes \\ 1, rue de la Noë, 44321 Nantes, France \\ Damien.Chablat@lan.ec-nantes.fr Philippe.Wenger@lan.ec-nantes.fr

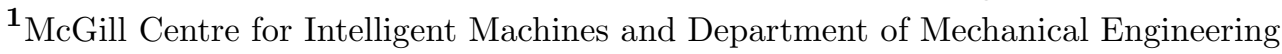 \\ McGill University, 817 Sherbrooke Street West \\ Montreal, Quebec, Canada H3A 2K6 \\ Angeles@cim.mcgill.ca
}

\begin{abstract}
The subject of this paper is a special class of closedchain manipulators. First, we analyze a family of twodegree-of-freedom (dof) five-bar planar linkages. Two Jacobian matrices appear in the kinematic relations between the joint-rate and the Cartesian-velocity vectors, which are called the "inverse kinematics" and the "direct kinematics" matrices. It is shown that the loci of points of the workspace where the condition number of the direct-kinematics matrix remains constant, i.e., the isoconditioning loci, are the coupler points of the four-bar linkage obtained upon locking the middle joint of the linkage. Furthermore, if the line of centers of the two actuated revolutes is used as the axis of a third actuated revolute, then a three-dof hybrid manipulator is obtained. The isoconditioning loci of this manipulator are surfaces of revolution generated by the isoconditioning curves of the two-dof manipulator, whose axis of symmetry is that of the third actuated revolute.

KEY WORDS : Kinematics, Closed-Loop Manipulator, Hybrid manipulator, Isoconditioning surfaces, Singularity, Working Modes.
\end{abstract}

\section{Introduction}

The aim of this paper is to study (a) a family of two-dof, five-bar planar linkages and (b) a derivative of this family, obtained when a third revolute is added in series to the above linkages, with the purpose of obtaining a three-dof manipulator. For the mechanical design of this class of manipulators, various features must be considered, e.g., the workspace volume, manipulability, and stiffness. The analysis of single- dof closed-loop chains is classical within the theory of machines and mechanisms [1]. The study of the workspace and the mobility of closed-loop manipulators, in turn, is given by Bajpai and Roth [2]. Gosselin [3], [4] conducted similar analyses for closed-loop manipulators with one single inverse kinematic solution on both a planar and a spatial mechanism. One important property of parallel manipulators is that they admit several solutions to both their inverse and their direct kinematics. This property leads to two types of singularities.

The singularities of these manipulators are correspondingly associated with two Jacobian matrices called here the "inverse kinematics" and the "direct kinematics" matrices. By means of the inverse kinematics matrix, we can define the "working mode" of the manipulator to separate the inverse kinematics solutions. It is useful to represent the manipulator in the workspace and to define its aspects in this workspace. The aspects of a manipulator are defined in [5]. Moreover, a novel three-dof hybrid manipulator is proposed, which is comparable to the one proposed by Bajpai and Roth 2]; ours is obtained as the series array of a one-revolute chain and the two-dof closedchain manipulator described above. In this array, the axis of the former intersects the axes of the two actuated joints of the latter at right angles.

The proper operation of a manipulator depends first of foremost on its design; besides design, the operation depends on suitable trajectory-planning and control algorithms. In any event, a performance index needs be defined, whose minimization or maximization leads to an optimum operation. While various items come into play when assessing the operation of a manipulator, we focus here on issues pertaining to 
manipulability or dexterity. In this regard, we understand these terms in the sense of measures of distance to singularity, which brings us to the concept of condition number [6]. Here, we adopt the condition number of the underlying Jacobian matrices as a means to quantify distances to singularity. Furthermore, we derive the loci of points of the joint and Cartesian workspaces whereby the condition number of each of the Jacobian matrices remains constant. For the planar two-dof manipulators studied here, we term these loci the isoconditioning curves, while, for threedof spatial manipulators, these curves become the isoconditioning surfaces.

\section{A Two-DOF Closed-Chain Manipu- lator}

The manipulator under study is a five-bar, revolute $(R)$-coupled linkage, as displayed in Fig. 1. The actuated joint variables are $\theta_{1}$ and $\theta_{2}$, while the Cartesian variables are the $(x, y)$ coordinates of the revolute center $P$.

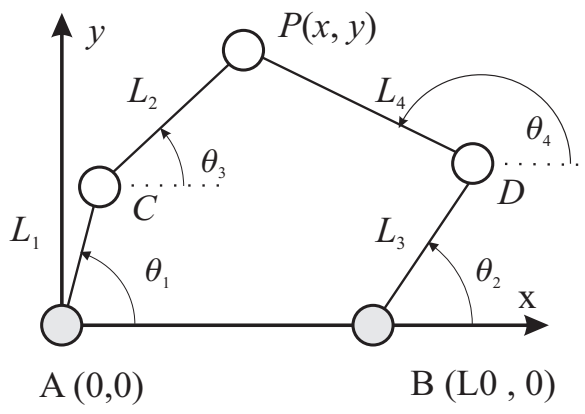

Figure 1: A two-dof closed-chain manipulator

Lenghts $L_{0}, L_{1}, L_{2}, L_{3}$, and $L_{4}$ define the geometry of this manipulator entirely. However, in this paper we focus on a symmetric manipulators, with $L_{1}=L_{3}$ and $L_{2}=L_{4}$. The symmetric architecture of the manipulator at hand is justified for general tasks. In manipulator design, then, one is interested in obtaining values of $L_{0}, L_{1}$, and $L_{2}$ that optimize a given objective function under some prescribed constraints.

\subsection{Kinematic Relations}

The velocity $\dot{\mathbf{p}}$ of point $P$, of position vector $\mathbf{p}$, can be obtained in two different forms, depending on the direction in which the loop is traversed, namely,

$$
\dot{\mathbf{p}}=\dot{\mathbf{c}}+\dot{\theta}_{3} \mathbf{E}(\mathbf{p}-\mathbf{c})
$$

$$
\dot{\mathbf{p}}=\dot{\mathbf{d}}+\dot{\theta}_{4} \mathbf{E}(\mathbf{p}-\mathbf{d})
$$

with matrix $\mathbf{E}$ defined as

$$
\mathbf{E}=\left[\begin{array}{cc}
0 & -1 \\
1 & 0
\end{array}\right]
$$

and $\mathbf{c}$ and $\mathbf{d}$ denoting the position vectors, in the frame indicated in Fig. 1, of points $C$ and $D$, respectively.

Furthermore, note that $\dot{\mathbf{c}}$ and $\dot{\mathbf{d}}$ are given by

$$
\dot{\mathbf{c}}=\dot{\theta}_{1} \mathbf{E} \mathbf{c}, \quad \dot{\mathbf{d}}=\dot{\theta}_{2} \mathbf{E}(\mathbf{d}-\mathbf{b})
$$

We would like to eliminate the two idle joint rates $\dot{\theta}_{3}$ and $\dot{\theta}_{4}$ from eqs.(1a) and (1b), which we do upon dotmultiplying the former by $\mathbf{p}-\mathbf{c}$ and the latter by $\mathbf{p}-\mathbf{d}$, thus obtaining

$$
\begin{aligned}
& (\mathbf{p}-\mathbf{c})^{T} \dot{\mathbf{p}}=(\mathbf{p}-\mathbf{c})^{T} \dot{\mathbf{c}} \\
& (\mathbf{p}-\mathbf{d})^{T} \dot{\mathbf{p}}=(\mathbf{p}-\mathbf{d})^{T} \dot{\mathbf{d}}
\end{aligned}
$$

Equations (2a) and (2b) can now be cast in vector form, namely,

$$
\mathbf{A} \dot{\mathbf{p}}=\mathbf{B} \dot{\theta}
$$

with $\dot{\boldsymbol{\theta}}$ defined as the vector of actuated joint rates, of components $\dot{\theta}_{1}$ and $\dot{\theta}_{2}$. Moreover $\mathbf{A}$ and $\mathbf{B}$ are, respectively, the direct-kinematics and the inversekinematics matrices of the manipulator, defined as

$$
\mathbf{A}=\left[\begin{array}{c}
(\mathbf{p}-\mathbf{c})^{T} \\
(\mathbf{p}-\mathbf{d})^{T}
\end{array}\right]
$$

and

$$
\mathbf{B}=L_{1} L_{2}\left[\begin{array}{cc}
\sin \left(\theta_{3}-\theta_{1}\right) & 0 \\
0 & \sin \left(\theta_{4}-\theta_{2}\right)
\end{array}\right]
$$

\section{The Isoconditioning Curves}

We derive below the loci of equal condition number of the direct- and inverse-kinematics matrices. To do this, we first recall the definition of condition number of an $m \times n$ matrix $\mathbf{M}$, with $m \leq n, \kappa(\mathbf{M})$. This number can be defined in various ways; for our purposes, we define $\kappa(\mathbf{M})$ as the ratio of the largest, $\sigma_{l}$, to the smallest $\sigma_{s}$, singular values of $\mathbf{M}$, namely,

$$
\kappa(\mathbf{M})=\frac{\sigma_{l}}{\sigma_{s}}
$$

The singular values $\left\{\sigma_{k}\right\}_{1}^{m}$ of matrix $\mathbf{M}$ are defined, in turn, as the square roots of the nonnegative eigenvalues of the positive-semidefinite $m \times m$ matrix $\mathbf{M M}^{\mathbf{T}}$. 


\subsection{Direct-Kinematics Matrix}

To calculate the condition number of matrix $\mathbf{A}$, we need the product $\mathbf{A} \mathbf{A}^{T}$, which we calculate below:

$$
\mathbf{A} \mathbf{A}^{T}=L_{2}^{2}\left[\begin{array}{cc}
1 & \cos \left(\theta_{3}-\theta_{4}\right) \\
\cos \left(\theta_{3}-\theta_{4}\right) & 1
\end{array}\right]
$$

The eigenvalues $\alpha_{1}$ and $\alpha_{2}$ of the above product are given by:

$$
\alpha_{1}=1-\cos \left(\theta_{3}-\theta_{4}\right), \quad \alpha_{2}=1+\cos \left(\theta_{3}-\theta_{4}\right)
$$

and hence, the condition number of matrix $\mathbf{A}$ is

$$
\kappa(\mathbf{A})=\sqrt{\frac{\alpha_{\max }}{\alpha_{\min }}}
$$

where

$$
\alpha_{\min }=1-\left|\cos \left(\theta_{3}-\theta_{4}\right)\right|, \quad \alpha_{\max }=1+\left|\cos \left(\theta_{3}-\theta_{4}\right)\right|
$$

Upon simplification,

$$
\kappa(\mathbf{A})=\frac{1}{\left|\tan \left(\left(\theta_{3}-\theta_{4}\right) / 2\right)\right|}
$$

In light of expression (9) for the condition number of the Jacobian matrix $\mathbf{A}$, it is apparent that $\kappa(\mathbf{A})$ attains its minimum of 1 when $\left|\theta_{3}-\theta_{4}\right|=\pi / 2$, the equality being understood modulo $\pi$. At the other end of the spectrum, $\kappa(\mathbf{A})$ tends to infinity when $\theta_{3}-\theta_{4}=k \pi$, for $k=1,2, \ldots$. When matrix $\mathbf{A}$ attains a condition number of unity, it is termed isotropic, its inversion being performed without any roundoff-error amplification. Manipulator postures for which condition $\theta_{3}-\theta_{4}=\pi / 2$ holds are thus the most accurate for purposes of the direct kinematics of the manipulator. Correspondingly, the locus of points whereby matrix $\mathbf{A}$ is isotropic is called the isotropy locus in the Cartesian workspace.

On the other hand, manipulator postures whereby $\theta_{3}-\theta_{4}=k \pi$ denote a singular matrix A. Such singularities occur at the boundary of the Joint space of the manipulator, and hence, the locus of $P$ whereby these singularities occur, namely, the singularity locus in the Joint space, defines this boundary. Interestingly, isotropy can be obtained regardless of the dimensions of the manipulator, as long as $i$ ) it is symmetric and $i i) L_{2} \neq 0$.

\subsection{Inverse-Kinematics Matrix}

By virtue of the diagonal form of matrix $\mathbf{B}$, its singular values, $\beta_{1}$ and $\beta_{2}$, are simply the absolute values of its diagonal entries, namely,

$$
\beta_{1}=\left|\sin \left(\theta_{3}-\theta_{1}\right)\right|, \quad \beta_{2}=\left|\sin \left(\theta_{4}-\theta_{2}\right)\right|
$$

The condition number $\kappa$ of matrix $\mathbf{B}$ is thus

$$
\kappa(\mathbf{B})=\sqrt{\frac{\beta_{\max }}{\beta_{\min }}}
$$

where, if $\left|\sin \left(\theta_{3}-\theta_{1}\right)\right|<\left|\sin \left(\theta_{4}-\theta_{2}\right)\right|$, then

$$
\beta_{\min }=\left|\sin \left(\theta_{3}-\theta_{1}\right)\right|, \quad \beta_{\max }=\left|\sin \left(\theta_{4}-\theta_{2}\right)\right| ;
$$

else,

$$
\beta_{\min }=\left|\sin \left(\theta_{4}-\theta_{2}\right)\right|, \quad \beta_{\max }=\left|\sin \left(\theta_{3}-\theta_{1}\right)\right| .
$$

In light of expression (11) for the condition number of the Jacobian matrix $\mathbf{B}$, it is apparent that $\kappa(\mathbf{B})$ attains its minimum of 1 when $\left|\sin \left(\theta_{3}-\theta_{1}\right)\right|=$ $\left|\sin \left(\theta_{4}-\theta_{2}\right)\right| \neq 0$. The locus of points where $\kappa(\mathbf{B})=$ 1 , and hence, where $\mathbf{B}$ is isotropic, is called the isotropy locus of the manipulator in the joint space. At the other end of the spectrum, $\kappa(\mathbf{B})$ tends to infinity when $\left|\theta_{3}-\theta_{1}\right|=k \pi$ or $\left|\theta_{4}-\theta_{2}\right|=k \pi$, for $k=1,2, \ldots$, which denote singularities of $\mathbf{B}$. These singularities are associated with the inverse kinematics of the manipulator, and hence, lie within its Cartesian workspace, not at the boundary of this one. The singularity locus of $\mathbf{B}$ thus defines the Cartesian workspace of the manipulator. Therefore, the Cartesian workspace of the manipulator is bounded by the singularity locus of $\mathbf{B}$, i.e., the locus of points where $\kappa(\mathbf{B}) \rightarrow \infty$. Interestingly, $\mathbf{B}$ can be rendered isotropic regardless of the dimensions of the manipulator, as long as $i$ ) it is symmetric and $i i) L_{1} \neq 0$ and $L_{2} \neq 0$.

\subsection{The Working Mode}
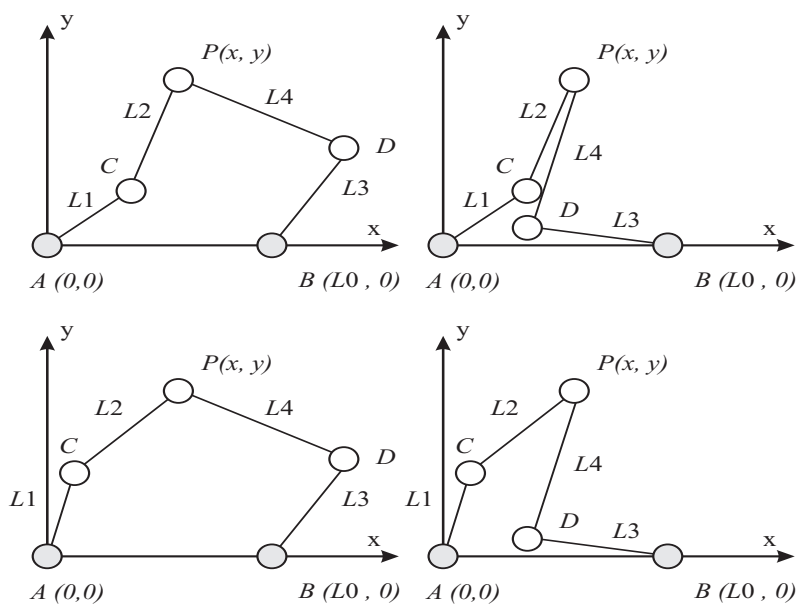

Figure 2: The four working modes 
The manipulator under study has a diagonal inverse-kinematics matrix $\mathbf{B}$, as shown in eq.(3d), the vanishing of one of its diagonal entries thus indicating the occurrence of a serial singularity. The set of manipulator postures free of this kind of singularity is termed a working mode. The different working modes are thus separated by a serial singularity, with a set of postures in different working modes corresponding to an inverse kinematics solution.

The formal definition of the working mode is detailed in [5]. For the manipulator at hand, there are four working modes, as depicted in Fig. 2.

\subsection{Examples}

We assume here the dimensions $L_{0}=6, L_{1}=8$, and $L_{2}=5$, in certain units of length that we need not specify.

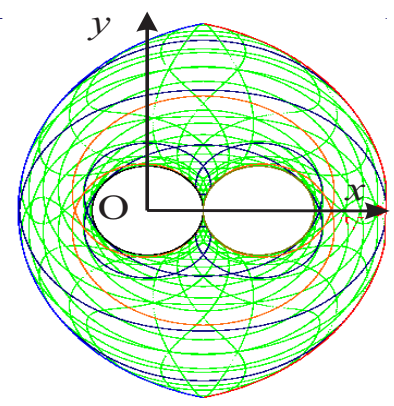

Figure 3: The isoconditioning curves in the Cartesian space

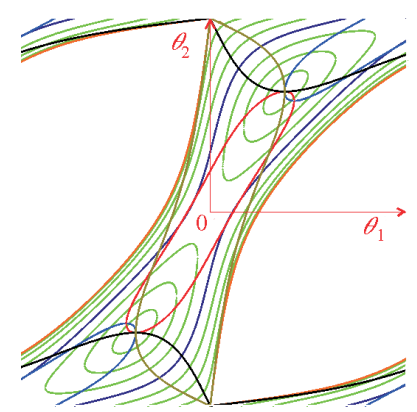

Figure 4: The isoconditioning curves in the joint space
The isoconditioning curves for the direct-kinematic matrix both in the Cartesian and in the joint spaces are displayed in Figs. 3 and 4 , respectively. A better representation of isoconditioning curves can be obtained in the Cartesian space by displaying these curves for every working mode, which we do in Fig. 5 .

In this figure, the isoconditioning curves are the coupler curves of the four-bar linkage derived upon locking the middle joint, of center $P(x, y)$, to yield a fixed value of $\theta_{3}-\theta_{4}$. Each configuration where points $C$ and $D$ coincide leads to a singularity where the position of point $P$ is not controllable.

\section{A Three-DOF Hybrid Manipulator}

Now we add one-dof to the manipulator of Fig. 1. We do this by allowing the overall two-dof manipula-
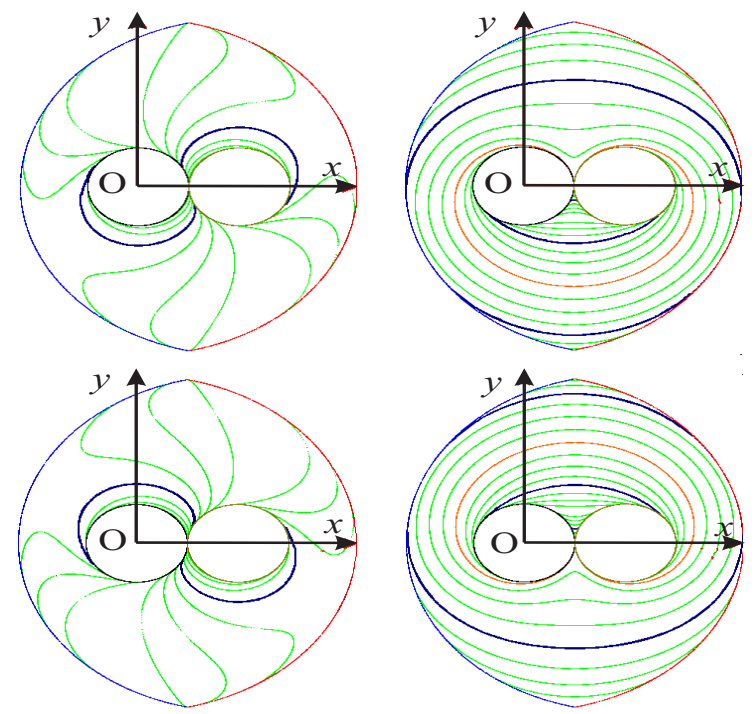

Figure 5: The four working modes and their isoconditioning curves in the Cartesian space

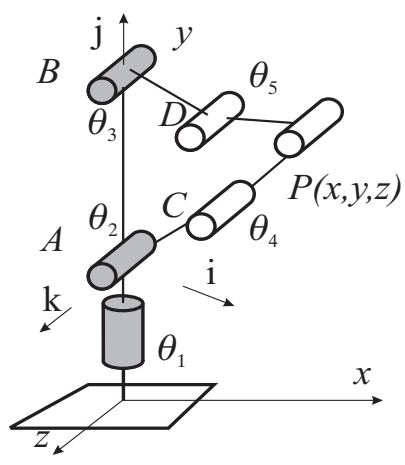

Figure 6: The three-dof hybrid manipulator

tor to rotate about line $A B$ by means of a revolute coupling the fixed link of the above manipulator with the base of the new manipulator. We thus obtain the manipulator of Fig. 6.

\subsection{Kinematic Relations}

The velocity $\dot{\mathbf{p}}$ of point $P$ can be obtained in two different forms, depending on the direction in which the loop is traversed, namely,

$$
\dot{\mathbf{p}}=\dot{\mathbf{c}}+\left(\dot{\theta}_{1} \mathbf{j}+\dot{\theta}_{4} \mathbf{k}\right) \times(\mathbf{p}-\mathbf{c})
$$

and

$$
\dot{\mathbf{p}}=\dot{d}+\left(\dot{\theta}_{1} \mathbf{j}+\dot{\theta}_{5} \mathbf{k}\right) \times(\mathbf{p}-\mathbf{d})
$$

Upon dot-multiplying eq.(14a) by $(\mathbf{p}-\mathbf{c})$ and eq. $14 \mathrm{~b})$ by $(\mathbf{p}-\mathbf{d})$, we obtain two scalar equations 
free of $\dot{\theta}_{1}$ and the idle joint rates $\dot{\theta}_{4}$ and $\dot{\theta}_{5}$, i.e.,

$$
\begin{aligned}
(\mathbf{p}-\mathbf{c})^{T} \dot{\mathbf{p}} & =(\mathbf{p}-\mathbf{c})^{T} \dot{\mathbf{c}} \\
(\mathbf{p}-\mathbf{d})^{T} \dot{\mathbf{p}} & =(\mathbf{p}-\mathbf{d})^{T} \dot{\mathbf{d}}
\end{aligned}
$$

Furthermore, we note that $\dot{\mathbf{c}}$ and $\dot{\mathbf{d}}$ are given by

$$
\begin{aligned}
\dot{\mathbf{c}} & =\left(\dot{\theta}_{1} \mathbf{j}+\dot{\theta}_{2} \mathbf{k}\right) \times \mathbf{c} \\
\dot{\mathbf{d}} & =\left(\dot{\theta}_{1} \mathbf{j}+\dot{\theta}_{3} \mathbf{k}\right) \times(\mathbf{d}-\mathbf{b})
\end{aligned}
$$

Substitution of the above two equations into eqs. 15 \& 16), two kinematic relations between joint rates and Cartesian velocities are obtained, namely,

$$
\begin{aligned}
{[(\mathbf{p}-\mathbf{c}) \times \mathbf{c}] \cdot \mathbf{k} \dot{\theta}_{2} } & =(\mathbf{p}-\mathbf{c})^{T} \dot{\mathbf{p}} \\
{[(\mathbf{p}-\mathbf{d}) \times(\mathbf{d}-\mathbf{b})] \cdot \mathbf{k} \dot{\theta}_{3} } & =(\mathbf{p}-\mathbf{d})^{T} \dot{\mathbf{p}}
\end{aligned}
$$

Moreover, upon dot-multiplying eqs.(14a \& b) by $\mathbf{k}$, we obtain two expressions for the projection of $\dot{\mathbf{p}}$ onto the $Z$ axis

$$
\begin{aligned}
\mathbf{k}^{T} \dot{\mathbf{p}} & =\mathbf{k}^{T}\left[\dot{\mathbf{c}}+\dot{\theta}_{1} \mathbf{j} \times(\mathbf{p}-\mathbf{c})\right] \\
\mathbf{k}^{T} \dot{\mathbf{p}} & =\mathbf{k}^{T}\left[\dot{\mathbf{d}}+\dot{\theta}_{1} \mathbf{j} \times(\mathbf{p}-\mathbf{d})\right]
\end{aligned}
$$

which, in light of eqs. (17 \& 18), readily reduce to

$$
\begin{aligned}
& \mathbf{k}^{T} \dot{\mathbf{p}}=\mathbf{i}^{\mathbf{T}} \mathbf{p} \dot{\theta}_{1} \\
& \mathbf{k}^{T} \dot{\mathbf{p}}=\mathbf{i}^{\mathbf{T}} \mathbf{p} \dot{\theta}_{1}
\end{aligned}
$$

It is apparent that the right-hand sides of the two foregoing equations are identical, and hence, those two scalar equations lead to exactly the same relation, namely,

$$
\mathbf{k}^{T} \dot{\mathbf{p}}=\left(\mathbf{i}^{\mathbf{T}} \mathbf{p}\right) \dot{\theta}_{1}
$$

It will prove useful to have the two sides of the above equation multiplied by $L_{2}$, and hence, that equation is equivalent to

$$
L_{2} \mathbf{k}^{T} \dot{\mathbf{p}}=L_{2}\left(\mathbf{i}^{T} \mathbf{p}\right) \dot{\theta}_{1}
$$

In the next step, we assemble eqs.(19) \& 20), which leads to an equation formally identical to eq.(3a), but with $\mathbf{A}$ and $\mathbf{B}$ defined now as $3 \times 3$ matrices, i.e.,

$$
\begin{aligned}
& \mathbf{A} \equiv\left[\begin{array}{c}
L_{2} \mathbf{k}^{T} \\
(\mathbf{p}-\mathbf{c})^{T} \\
(\mathbf{p}-\mathbf{d})^{T}
\end{array}\right] \\
& \mathbf{B} \equiv L_{1} L_{2}\left[\begin{array}{rrr}
\sin \theta_{2}+\lambda_{1} \sin \theta_{4} & 0 & 0 \\
0 & \sin \left(\theta_{2}-\theta_{4}\right) & 0 \\
0 & 0 & \sin \left(\theta_{3}-\theta_{5}\right)
\end{array}\right]
\end{aligned}
$$

with $\lambda_{1}$ defined as $\lambda_{1} \equiv L_{2} / L_{1}$, while vectors $\dot{\boldsymbol{\theta}}$ and $\dot{\mathbf{p}}$ are now given by

$$
\dot{\boldsymbol{\theta}} \equiv\left[\begin{array}{c}
\dot{\theta}_{1} \\
\dot{\theta}_{2} \\
\dot{\theta}_{3}
\end{array}\right], \quad \dot{\mathbf{p}} \equiv\left[\begin{array}{c}
\dot{x} \\
\dot{y} \\
\dot{z}
\end{array}\right]
$$

\section{The Isoconditioning Surfaces}

We conduct here the same analysis of Section 3 .

\subsection{The Direct-Kinematics Matrix}

Apparently, matrix $\mathbf{A}$ in the 3-dof case has a structure similar to the corresponding matrix in the 2-dof case. Indeed, upon calculating $\mathbf{A} \mathbf{A}^{T}$ in the 3 -dof case, we obtain

$$
\mathbf{A A}^{T}=L_{2}^{2}\left[\begin{array}{ccc}
1 & 0 & 0 \\
0 & 1 & \cos \left(\theta_{4}-\theta_{5}\right) \\
0 & \cos \left(\theta_{4}-\theta_{5}\right) & 1
\end{array}\right]
$$

The eigenvalues of the foregoing matrix are, then, $\alpha_{1}=1-\left|\cos \left(\theta_{4}-\theta_{5}\right)\right|, \alpha_{2}=1$, and $\alpha_{3}=1+\mid \cos \left(\theta_{4}-\right.$ $\left.\theta_{5}\right) \mid$, the foregoing eigenvalues having been ordered as

$$
\alpha_{1} \leq \alpha_{2} \leq \alpha_{3}
$$

The condition number of matrix $\mathbf{A}$ is thus

$$
\kappa(\mathbf{A})=\sqrt{\frac{1+\left|\cos \left(\theta_{4}-\theta_{5}\right)\right|}{1-\left|\cos \left(\theta_{4}-\theta_{5}\right)\right|}}
$$

which can be further simplified to

$$
\kappa(\mathbf{A})=\frac{1}{\left|\tan \left(\left(\theta_{4}-\theta_{5}\right) / 2\right)\right|}
$$

Therefore, the condition number of the two directkinematics matrices, for the 2-dof and the 3-dof cases, coincide. However, the loci of isoconditioning points are now surfaces, because we have added one dof to the manipulator of Fig. 1. These loci are, in fact, surfaces of revolution generated by the isoconditioning curves of the 2-dof manipulator, when these are rotated about the axis of the first revolute. We represent the boundary of the workspace (Fig. 耳).

\subsection{The Inverse-Kinematics Matrix}

Given the diagonal structure of matrix $\mathbf{B}$, its singular values are apparently, $\left\{L_{1} L_{2} \beta_{i}\right\}_{1}^{3}$, with the definitions below:

$$
\begin{aligned}
& \beta_{1}=\left|\sin \theta_{2}+\lambda_{1} \sin \theta_{4}\right|, \\
& \beta_{2}=\left|\sin \left(\theta_{2}-\theta_{4}\right)\right|, \\
& \beta_{3}=\left|\sin \left(\theta_{3}-\theta_{5}\right)\right|
\end{aligned}
$$

Therefore, the isoconditioning locus of $\mathbf{B}$ is determined by the relation

$$
\left|\sin \theta_{2}+\lambda_{1} \sin \theta_{4}\right|=\left|\sin \left(\theta_{2}-\theta_{4}\right)\right|=\left|\sin \left(\theta_{3}-\theta_{5}\right)\right|
$$




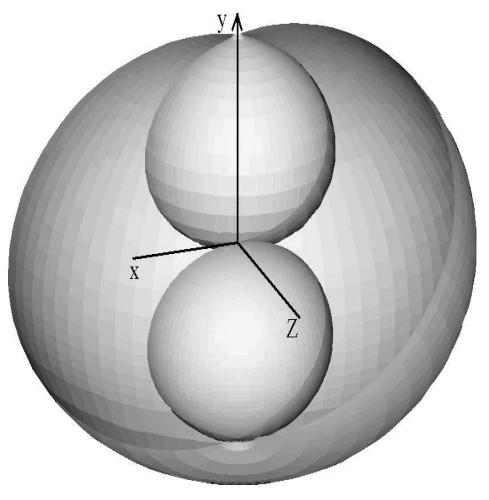

Figure 7: The boundary of the workspace

Notice that the distance $d_{1}$ of $P$ to the $Y$ axis is

$$
d_{1}=L_{1} \sin \theta_{2}+L_{2} \sin \theta_{4}=L_{1} \beta_{1}
$$

Likewise, the distances $d_{2}$ and $d_{3}$ of $P$ to the two axes of the other two actuated revolutes, i.e., those passing through $A$ and $B$ are, respectively,

$$
\begin{aligned}
& d_{2}=L_{2} \beta_{2} \\
& d_{3}=L_{2} \beta_{3}
\end{aligned}
$$

It is now straightforward to realize that, for the case at hand, the locus of isotropic points of $\mathbf{B}$ are given by manipulator postures whereby $P$ is equidistant from the three actuated revolute axes. Likewise, postures whereby point $P$ lies on the $Y$ axis are singular; at these postures, $\kappa(\mathbf{B})$ tends to infinity. Moreover, the inverse-kinematics singularities occur whenever any of the diagonal entries of $\mathbf{B}$ vanishes, i.e., when

$$
d_{1}=0, \text { or } \theta_{2}=\theta_{4}+k \pi, \text { or } \theta_{3}=\theta_{5}+k \pi
$$

for $k=1,2, \ldots$

\section{Conclusions}

We have defined a new architecture of hybrid manipulators and derived the associated loci of isoconditioning points. Two Jacobian matrices were identified in the mapping of joint rates into Cartesian velocities, namely, the direct-kinematics and the inversekinematics matrices. Isoconditioning loci were defined for these matrices. Two special loci were discussed, namely, those pertaining to isotropy and to singularity, for each of these matrices.

The study has been conducted for three-dof-hybrid manipulators but applies to six-dof-hybrid manipulators with wrist as well.
The hybrid manipulators studied have interesting features like workspace and high dynamic performances, which are usually met separately in serial or parallel manipulators, respectively. Futher research work is being conducted by the authors on such hybrid manipulators with regard to their optimal design.

\section{Acknowledgments}

The third author acknowledges the support from the Natural Sciences and Engineering Research Council, of Canada, the Fonds pour la formation de chercheurs et l'aide à la recherche, of Quebec, and École Centrale de Nantes (ECN). The research reported here was conducted during a sojourn that this author spent at ECN's Institut de Recherche en $\mathrm{Cy}$ bernétique de Nantes.

\section{References}

[1] Hunt, K. H. "Geometry of Mechanisms" Clarendon Press, Oxford, 1978.

[2] Bajpai, A. and Roth, B. "Workspace and mobility of a closed-loop manipulator" The International Journal of Robotics Research, Vol. 5, No. 2, 1986.

[3] Gosselin, C. "Stiffness mapping for parallel manipulators" IEEE Transactions On Robotics And Automation, Vol. 6, No. 3, June 1990.

[4] Gosselin, C. and Angeles, J. "Singularity analysis of closed-loop kinematic chains" IEEE Transactions On Robotics And Automation, Vol. 6, No. 3, June 1990 .

[5] Chablat, D. and Wenger, Ph. "Working modes and aspects in fully parallel manipulators" to appear in Proc. IEEE International Conference of Robotic and Automation, Mai 1998.

[6] Golub, G. H. and Van Loan, C. F. "Matrix Computations" The Johns Hopkins University Press, Baltimore, 1989. 